\title{
Corrections
}

The paper

\section{Preferred orientation of the internal structure of carbon layers in carbon fibers}

by R. Plaetschke and W. Ruland

which appeared in Progr Coll \& Polym Sci 71:140-144 (1985)

has been printed with mistakes in Table 2, which we should like to correct as follows:

Table 2. $a$-axis and $c$-axis orientation parameters and type of orientation

\begin{tabular}{llll}
\hline Sample & $q_{a}$ & $\left|q_{c}\right|$ & $\begin{array}{l}\text { Type of } \\
\text { orientation }\end{array}$ \\
\hline M1 & 0 & 0.49 & - \\
M2 & 0.30 & 0.90 & 0 \\
M3 & 0.33 & 0.92 & 0 \\
M4 & 0.41 & 0.93 & 0 \\
P1 & -0.30 & 0.82 & 0 \\
P2 & -0.27 & 0.83 & 0 \\
P3 & -0.19 & 0.87 & 0 \\
P4 & 0 & 0.62 & - \\
P5 & -0.27 & 0.82 & 0 \\
& & & \\
C1 & 0 & 0.85 & - \\
C2 & 0 & 0.86 & - \\
C3 & 0.30 & 0.89 & 0 \\
C4 & 0.31 & 0.91 & 0 \\
\hline
\end{tabular}

Für die Schriftleitung verantwortlich: Für Originalarbeiten Prof. Dr. Hanns-Georg Kilian, Universität Ulm, Abt. Experimentelle Physik, Oberer Eselsberg, $7900 \mathrm{Ulm}$, und Prof. Dr. Dr. h. c. Armin Weiss, Institut für anorganische Chemie der Universität, Meiserstraße 1, 8000 München 2 - Anzeigenverwaltung: Springer-Verlag GmbH \& Co. KG, Heidelberger Platz 3, D-1000 Berlin 33 - Verantwortlich für den Anzeigenteil: E. Lückermann - Verlag: Dr. Dietrich Steinkopff Verlag GmbH \& Co. KG, Saalbaustraße 12, Postfach 11 10 08, D-6100 Darmstadt 11 - Herstellung: Meister Druck, 3500 Kassel 